\title{
High Prevalence of Inappropriate Benzodiazepine and Sedative Hypnotic Prescriptions among Hospitalized Older Adults
}

\author{
Elisabeth Anna Pek, MD¹, Andrew Remfry, MD¹, Ciara Pendrith, MSc², Chris Fan-Lun, BScPhm³, \\ R. Sacha Bhatia, $\mathrm{MD}^{1,4-6}$, Christine Soong, MD, MSc $\mathrm{M}^{6-8^{*}}$
}

\begin{abstract}
${ }^{1}$ Department of Medicine, University of Toronto, Ontario; 'Institute of Health System Solutions and Virtual Care, Women's College Hospital, Toronto, Ontario; ' ${ }^{2}$ eslie Dan Faculty of Pharmacy, University of Toronto, Ontario; ${ }^{4}$ Women's College Hospital Institute for Health Systems Solutions and

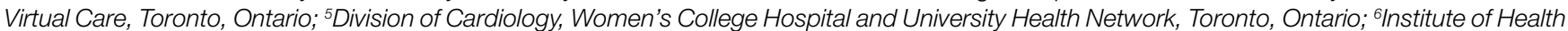
Policy Management and Evaluation, University of Toronto, Toronto, Ontario; 'Division of General Internal Medicine, Sinai Health System, Toronto, Ontario; ${ }^{8}$ Centre for Quality Improvement and Patient Safety, University of Toronto, Toronto, Ontario.
\end{abstract}

BACKGROUND: Benzodiazepines and sedative hypnotics are commonly used to treat insomnia and agitation in older adults despite significant risk. A clear understanding of the extent of the problem and its contributors is required to implement effective interventions.

OBJECTIVE: To determine the proportion of hospitalized older adults who are inappropriately prescribed benzodiazepines or sedative hypnotics, and to identify patient and prescriber factors associated with increased prescriptions.

DESIGN: Single-center retrospective observational study.

SETTING: Urban academic medical center.

PARTICIPANTS: Medical-surgical inpatients aged 65 or older who were newly prescribed a benzodiazepine or zopiclone.

MEASUREMENTS: Our primary outcome was the proportion of patients who were prescribed a potentially inappropriate benzodiazepine or sedative hypnotic. Potentially inappropriate indications included new prescriptions for insomnia or agitation/anxiety. We used a multivariable random-intercept logistic regression model to identify patient- and prescriber-level variables that were associated with potentially inappropriate prescriptions.
RESULTS: Of 1308 patients, 208 (15.9\%) received a potentially inappropriate prescription. The majority of prescriptions, 254 (77.4\%), were potentially inappropriate. Of these, most were prescribed for insomnia (222; 87.4\%) and during overnight hours $(159 ; 62.3 \%)$. Admission to a surgical or specialty service was associated with significantly increased odds of potentially inappropriate prescription compared to the general internal medicine service (odds ratio [OR], 6.61; $95 \%$ confidence interval [Cl], 2.70-16.17). Prescription by an attending physician or fellow was associated with significantly fewer prescriptions compared to first-year trainees (OR, 0.28; 95\% Cl, 0.08-0.93). Nighttime prescriptions did not reach significance in initial bivariate analyses but were associated with increased odds of potentially inappropriate prescription in our regression model (OR, 4.48; $95 \% \mathrm{Cl}$, 2.21-9.06).

CONCLUSIONS: The majority of newly prescribed benzodiazepines and sedative hypnotics were potentially inappropriate and were primarily prescribed as sleep aids. Future interventions should focus on the development of safe sleep protocols and education targeted at first-year trainees. Journal of Hospital Medicine 2017;12:310-316. (C) 2017 Society of Hospital Medicine
Older adults commonly experience insomnia and agitation during hospitalization. Unfortunately, the use of benzodiazepines and sedative hypnotics (BSH) to treat these conditions can be ineffective and expose patients to significant adverse effects. ${ }^{1,2}$ Choosing Wisely ${ }^{\circledR}$ is a campaign that promotes dialogue to reduce unnecessary medical tests, procedures, or treatments. This international campaign has highlighted BSHs as potentially harmful and has recommended against their use as first-line treatment of insomnia and agitation. ${ }^{3-5}$ Examples of harm with benzodiazepine use include cognitive impairment, impaired postural stability, and an increased in-

\footnotetext{
*Address for correspondence and reprint requests: Christine Soong, MD, Mount Sinai Hospital, 428-600 University Avenue, Toronto, ON M5G 1X5; Telephone: 416-546-4800 x5464; Fax, 647-776-3184; E-mail: christine.soong@ utoronto.ca

Received: May 24, 2016; Revised: October 19, 2016; Accepted: November 1,2016
}

2017 Society of Hospital Medicine DOI 10.12788/jhm.2739 cidence of falls and hip fractures in both community and acute care settings. ${ }^{6-8}$ In addition, prescriptions initiated in hospital appear to be associated with a higher risk of falls and unplanned readmission., ${ }^{9,10}$ The newer nonbenzodiazepine sedative hypnotics, commonly referred to as "z-drugs", were initially marketed as a safer alternative in older adults due to their more favorable pharmacokinetics. Evidence has emerged that they carry similar risks. ${ }^{6,11,12} \mathrm{~A}$ study comparing benzodiazepines and zolpidem found relatively greater risk of fractures requiring hospitalization with the use of zolpidem compared to lorazepam. ${ }^{13}$

The use of benzodiazepines in the acute care setting has been evaluated in a number of studies and ranges from $20 \%$ to $45 \%{ }^{14-16} \mathrm{Few}$ studies focus on the initiation of these medications in BSH-naïve hospitalized patients; however, reports range from $18 \%$ to $29 \% .{ }^{17,18}$ Factors found to be associated with potentially inappropriate prescriptions (PIP) include Hispanic ethnicity, residing in an assisted care setting, and a greater number of $\mathrm{BSH}$ prescriptions prior to admission. ${ }^{16,19}$ 
Additionally, Cumbler et al. ${ }^{15}$ found that the presence of dementia was associated with fewer prescriptions for sleep aids in hospital. To our knowledge, there are no published studies that have investigated prescriber factors associated with the use of BSH.

The purpose of our study was to determine the frequency of PIPs of BSH in our academic hospital. Additionally, we aimed to identify patient and prescriber factors that were associated with increased likelihood of prescriptions to help guide future quality improvement initiatives.

\section{METHODS}

\section{Study Design and Setting}

This was a retrospective observational study conducted at Mount Sinai Hospital (MSH) in Toronto over a 4-month period from January 2013 to April 2013. The hospital is a 442-bed acute care academic health science center affiliated with the University of Toronto. The MSH electronic health record contains demographic data, medications and allergies, nursing documentation, and medical histories from prior encounters. It also includes computerized physician order entry $(\mathrm{CPOE})$ and a detailed medication administration record. This system is integrated with an electronic pharmacy database used to monitor and dispense medications for each patient.

\section{Patient and Medication Selection}

We included inpatients over the age of 65 who were prescribed a BSH during the study period from the following services: general internal medicine, cardiology, general surgery, orthopedic surgery, and otolaryngology. To identify new exposure to BSHs, we excluded patients who were regularly prescribed a BSH prior to admission to hospital. The medications of interest included all benzodiazepines and the nonbenzodiazepine sedative hypnotic, zopiclone. Zopiclone is the most commonly used nonbenzodiazepine sedative hypnotic in Canada and the only 1 available on our hospital formulary. These were selected based on the strength of evidence to recommend against their use as first-line agents in older adults and in consultation with our geriatric medicine consultation team pharmacist. ${ }^{20}$

\section{Data Collection}

The hospital administrative database provided patient demographic information, admission service, admitting diagnosis, length of stay, and the total number of patients discharged from the study units over the study period. We then searched the pharmacy electronic database for all benzodiazepines and zopiclone prescribed during the study period for patients who met the inclusion criteria. Manual review of paper and electronic health records for this cohort of patients was conducted to extract additional variables. We used a standardized form to record data elements. Dr. Pek collected all data elements. Dr. Remfry reviewed a random sample of patient records (10\%) to ensure accuracy. The agreement between reviewers was 100\%.
In compliance with hospital accreditation standards, a clinical pharmacist documents a best possible medication history (BPMH) on every inpatient on admission. We used the BPMH to identify and exclude patients who were prescribed a BSH prior to hospitalization. Because all medications were ordered through the CPOE system, as-needed medication prescriptions required the selection of a specified indication. Available options included 'agitation/anxiety' and necessitated combining these 2 indications into 1 category. Indications were primarily extracted through electronic order entry reviews. Paper charts were reviewed when further clarification was needed.

We identified ordering physicians' training level and familiarity with the service from administrative records obtained from medical education offices, hospital records, and relevant call schedules. Fellows were defined as trainees with a minimum of 6 years of postgraduate training.

\section{Variables}

Our primary outcome of interest was the proportion of eligible patients age 65 and older who received a PIP for a BSH. Patient variables of interest included age, sex, comorbid conditions, and a pre-admission diagnosis of dementia. Comorbid conditions and age were used to calculate the Carlson Comorbidity Index for each patient. ${ }^{21}$ Prescription variables included the medication prescribed, time of first prescription ("overnight hours" refer to prescriptions ordered after 7:00 PM and before 7:00 AM), and whether the medication was ordered as part of an admission or postoperative order set. To determine whether patients were discharged home with a prescription for a $\mathrm{BSH}$, we reviewed electronic discharge prescriptions of $\mathrm{BSH}$-naïve patients who received a sedative in hospital. Only medical and cardiology inpatients receive electronic discharge prescriptions, and these were available for 189 patients in our cohort. Provider variables included training level, service, and familiarity with patients. We used the provider's training program or department of appointment to define the 'physician on-service' variable. As an example, a resident registered in internal medicine is defined as 'on-service' when prescribing sedatives for a medical inpatient. In contrast, a psychiatry resident would be considered "off-service" if he prescribed a sedative for a surgical inpatient. The familiarity of a provider was categorized as 'regular' if they were responsible for a patient's care on a day-to-day basis and 'covering' if they were only covering on call. Other variables included admitting service and hospital length of stay.

\section{Appropriateness Criteria}

Criteria for potentially inappropriate use were modified from the American and Canadian Geriatrics Societies' Choosing Wisely recommendations, ${ }^{4,5}$ and included insomnia and agitation. These recommendations are in line with other evidence based guidelines for safe prescribing in older adults. ${ }^{20}$ For the purposes of our study, prescriptions for "agitation/ anxiety", "agitation", or "insomnia/sleep" were considered potentially inappropriate. Appropriate indications included 
TABLE 1. Characteristics of Study Patients Newly Prescribed a Benzodiazepine or Sedative Hypnotic In-Hospital

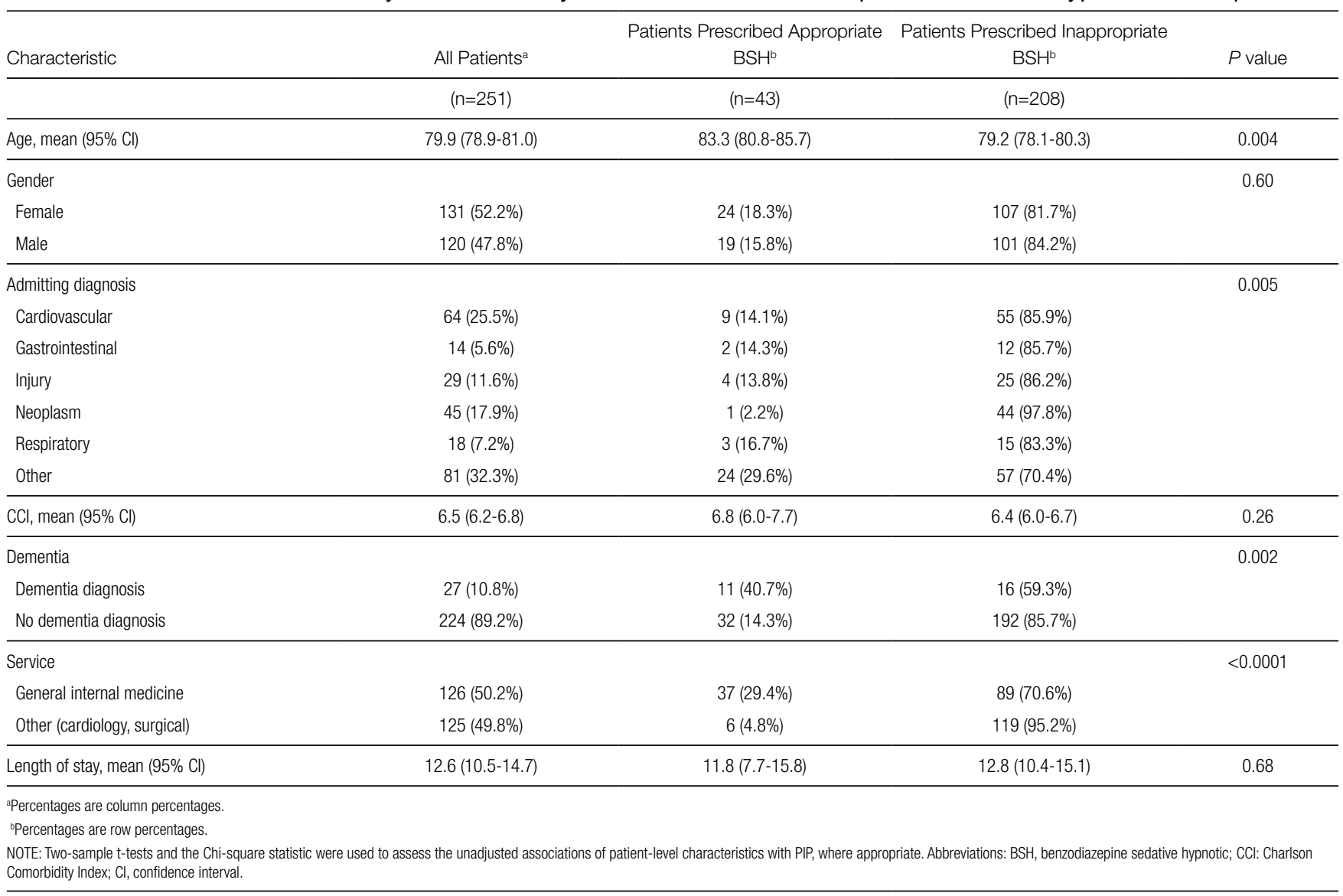

alcohol withdrawal, end-of-life symptom control, preprocedural sedation, and seizure. ${ }^{5}$ Patients who were already using a BSH prior to admission for any indication, including a psychiatric diagnosis, were excluded.

\section{Statistical Analyses}

We determined the proportion of patients with at least one PIP, as well as the proportion of all prescribing events that were potentially inappropriate. We used the Chi-square statistic and 2-sample $t$ tests to compare the unadjusted associations between patient-level characteristics and receipt of at least 1 inappropriate prescription and prescribing event-level factors with inappropriate prescriptions. Given that first-year residents are more likely to be working overnight when most PIPs are prescribed, we performed a simple logistic regression of potentially inappropriate prescribing by level of training stratified by time of prescription. A multivariable random-intercept logistic regression model was used to assess the adjusted association between patient- and prescribing event-level characteristics with inappropriate prescribing, adjusting for clustering of prescribing events within patients. Characteristics of interest were identified $a$ priori and those with significant bivariate associations with potentially inappropriate were selected for inclusion in the model. Additionally, we included time of prescription in our model to control for potential confounding. All statistical analyses were performed using SAS 9.4 (SAS Institute Inc, Cary, North Carolina). The MSH Research Ethics Board approved the study.

\section{RESULTS}

\section{Description of Patients Prescribed a Benzodiazepine Sedative Hypnotic}

There were 1540 patients over the age of 65 discharged during the 4-month study period. We excluded the $232 \mathrm{pa}$ tients who had been prescribed a BSH prior to admission. Of the remaining eligible $1308 \mathrm{BSH}$-naïve patients, 251 $(19.2 \%)$ were prescribed a new BSH in hospital and were included in the study. Of this cohort of 251 patients, 193 (76.9\%) patients were prescribed a single $\mathrm{BSH}$ during their admission while $58(23.1 \%)$ received 2 or more. Of all eligible patients, 208 (15.9\%) were prescribed at least 1 PIP. Approximately half of the cohort was admitted to the general internal medicine service, and the most common reason for admission was cardiovascular disease (Table 1).

\section{Description of Prescriptions of Benzodiazepine Sedative Hypnotic}

We reviewed 328 prescriptions for BSH during the study period. The majority of these, $254(77.4 \%)$ were potentially 
TABLE 2. Provider Characteristics of New In-Hospital Prescriptions and Timing of Prescribing Events

\begin{tabular}{|c|c|c|c|c|}
\hline Characteristic & All Prescriptions ${ }^{a}$ & Appropriate Prescriptions ${ }^{b}$ & Inappropriate Prescriptions ${ }^{b}$ & $P$ value \\
\hline & $(n=328)$ & $(n=74)$ & $(n=254)$ & \\
\hline Physician-training level & & & & 0.0007 \\
\hline PGY-1 & $213(64.9 \%)$ & $40(18.8 \%)$ & $173(81.2 \%)$ & \\
\hline PGY-2 & $36(11.0 \%)$ & $16(44.4 \%)$ & $20(55.6 \%)$ & \\
\hline PGY-3-5 & $53(16.2 \%)$ & $8(15.1 \%)$ & $45(84.9 \%)$ & \\
\hline Attending physician and fellows & $26(7.9 \%)$ & $10(38.5 \%)$ & $16(61.5 \%)$ & \\
\hline Physician service & & & & 0.076 \\
\hline On service & $225(68.6 \%)$ & $57(25.3 \%)$ & $168(74.7 \%)$ & \\
\hline Off service & 103 (31.4\%) & $17(16.5 \%)$ & $86(83.5 \%)$ & \\
\hline Physician familiarity & & & & 0.017 \\
\hline Regular & $260(79.3 \%)$ & $66(25.4 \%)$ & $194(74.6 \%)$ & \\
\hline Covering & $68(20.7 \%)$ & $8(11.8 \%)$ & $60(88.2 \%)$ & \\
\hline Time of prescription & & & & 0.067 \\
\hline Daytime & $150(45.7 \%)$ & $55(36.7 \%)$ & $95(63.3 \%)$ & \\
\hline Nighttime (7:00 PM to 7:00 AM) & 178 (54.3\%) & $19(10.7 \%)$ & $159(89.3 \%)$ & \\
\hline
\end{tabular}

inappropriate (Table 2). The most common PIPs were zopiclone $(167 ; 65.7 \%)$ and lorazepam $(82 ; 32.3 \%)$. The PIPs were most frequently ordered on an as-needed basis (219; $86 \%)$, followed by one-time orders $(30 ; 12 \%)$, and standing orders $(5 ; 2 \%)$. The majority of PIPs $(222 ; 87.4 \%)$ was prescribed for insomnia with a minority $(32 ; 12.6 \%)$ prescribed for agitation and/or anxiety.

Most PIP were prescribed during overnight hours (159; $62.6 \%)$ and when an in-house pharmacist was unavailable $(211 ; 83.1 \%)$. These variables were highly correlated with prescription of sleep aid, which was defined in our criteria as potentially inappropriate. Copies of discharge prescriptions were available for 189 patients. Of these 189 patients, $19(10.1 \%)$ were sent home with a prescription for a new sedative.

\section{Association Between Patient/Provider Variables and Prescriptions}

Patient factors associated with fewer PIPs in our bivariate analyses included older age and dementia (Table 1). A greater proportion of nighttime prescriptions were PIPs; however, this finding was not statistically significant $(P=0.067)$. The majority of all prescriptions was prescribed by residents in their first year of training (64.9\%; Table 2), and there was a significant difference in rates of PIP across level of training $(P=0.0007)$. When stratified by time of prescription, there was no significant difference by level of training for nighttime prescriptions. Among daytime prescriptions, second-year residents and staff (attending physicians and fellows) were less likely to prescribe a PIP than first-year residents (odds ratio [OR], 0.24; 95\% confidence interval [CI], 0.09-0.66 and OR, 0.39; 95\% CI, 0.14-1.13, respectively;
Table 3); however, the association between staff and firstyears only approached statistical significance $(P=0.08)$. Interestingly, $20.4 \%$ of all PIPs were ordered routinely as part of an admission or postoperative order set.

In our regression model, admission to a specialty or surgical service, compared to the general internal medicine service, was associated with a significantly higher likelihood of a PIP (OR, 6.61; 95\% CI, 2.70-16.17; Table 4). Additionally, compared to cardiovascular admission diagnoses, neoplastic admitting diagnoses were associated with a higher likelihood of a PIP (OR, 4.43; 95\% CI, 1.23-15.95). Time of prescription was a significant predictor in our multivariable regression model with nighttime prescriptions having increased odds of a PIP (OR, 4.48; 95\% CI, 2.21-9.06,). When comparing prescribers at the extremes of training, attending physicians and fellows were much less likely to prescribe a PIP compared to first-year residents (OR, 0.23; 95\% CI, 0.08-0.69; Table 4). However, there were no other significant differences across training levels after adjusting for patient and prescribing event characteristics.

\section{DISCUSSION}

We found that the majority of newly prescribed $\mathrm{BSH}$ in hospital was for the potentially inappropriate indications of insomnia and agitation/anxiety. Medications for insomnia were primarily initiated during overnight hours. Training level of prescribers and admitting service were found to be associated with appropriateness of prescriptions.

Our study showed that $15.9 \%$ of hospitalized older adults were newly prescribed a PIP during their admission. Of all new in hospital prescriptions, $77 \%$ were deemed potentially inappropriate. These numbers are similar to those reported 
TABLE 3. Associations Between Level of Training with Potentially Inappropriate Prescriptions of Benzodiazepines and Sedative Hypnotics Stratified by Timing of Prescription

\begin{tabular}{|c|c|c|c|c|}
\hline Timing of Prescription & Reference Level of Training & Comparison Level of Training & OR (95\% Cl) & $P$ value \\
\hline \multirow[t]{2}{*}{ Daytime (7:00 am to 7:00 PM) } & PGY-1 & PGY-2 & $0.24(0.09-0.66)$ & 0.0061 \\
\hline & & PGY-3-5 & $1.40(0.50-3.90)$ & 0.52 \\
\hline \multirow[t]{2}{*}{ Nighttime (7:00 pm to 7:00 AM) } & PGY-1 & PGY-2 & $0.50(0.13-2.00)$ & 0.33 \\
\hline & & PGY-3-5 & $1.51(0.32-7.10)$ & 0.61 \\
\hline
\end{tabular}

NOTE: Effect size estimates were calculated using simple logistic regression for the association between level of training with potentially inappropriate prescription stratified by timing of prescription. Abbreviations: $\mathrm{Cl}$, confidence interval; $\mathrm{OR}$, odds ratio

TABLE 4. Association of Patient and Prescription Characteristics with Potentially Inappropriate Prescriptions of Benzodiazepines and Sedative Hypnotics

\begin{tabular}{|c|c|c|c|c|}
\hline Characteristics & Reference Group & Comparison Group & OR $(95 \% \mathrm{Cl})$ & $P$ value \\
\hline \multirow[t]{2}{*}{ Admitting diagnosis } & \multirow[t]{2}{*}{ Circulatory } & Neoplasm & $4.43(1.23-15.95)$ & $0.023^{\mathrm{a}}$ \\
\hline & & Other & $1.60(0.64-3.95)$ & 0.31 \\
\hline Age, grouped & $65-79 y$ & $\geq 80 y$ & $1.05(0.50-2.21)$ & 0.90 \\
\hline Dementia & Dementia diagnosis & No dementia diagnosis & $1.79(0.67-4.83)$ & 0.25 \\
\hline Familiarity with patient & Regular & Covering & $1.22(0.42-3.57)$ & 0.72 \\
\hline Hospital service & General internal medicine & Other (surgical, cardiology) & $6.61(2.70-16.17)$ & $<0.001^{\mathrm{a}}$ \\
\hline Time of prescription & Daytime & Nighttime & $4.48(2.21-9.06)$ & $<0.001^{\mathrm{a}}$ \\
\hline \multirow[t]{3}{*}{ Training } & \multirow[t]{3}{*}{$P G Y-1$} & PGY-2 & $0.52(0.30-1.33)$ & 0.17 \\
\hline & & PGY-3-5 & $0.91(0.30-2.77)$ & 0.87 \\
\hline & & Attending physicians and fellows & $0.28(0.08-0.93)$ & $0.037^{\mathrm{a}}$ \\
\hline
\end{tabular}

aDenotes statistical significance at $P<0.05$.

NOTE: A multivariable random-intercept logistic regression model was used to assess the adjusted associations between patient- and prescribing event-level characteristics with inappropriate prescribing, adjusting for clustering of prescribing events within patients. Abbreviations: $\mathrm{Cl}$, confidence interval; OR, odds ratio; PGY, postgraduate year.

by other centers; however, wide ranges exist. ${ }^{16,19}$ This is likely the result of differences in appropriate use and inclusion criteria. Gillis et al. ${ }^{17}$ focused their investigation on sleep aids and showed that $26 \%$ of all admitted patients and $18 \%$ of BSH naïve patients received a prescription for insomnia. While this is similar to our findings, more than half of these patients were under the age of 65 , and additional medications, such as trazodone, antihistamines, and antipsychotics were included. ${ }^{17}$ Other studies did not exclude patients who used a BSH regularly prior to admission. For example, $21 \%$ of veterans admitted to an acute care facility received a prescription for potentially inappropriate indications, but this included continuation of prior home medications. ${ }^{19}$ In contrast, we chose to focus on older adults in whom BSH pose a greater risk of harm. Exclusion of patients who regularly used a BSH prior to admission allowed us to better understand the circumstances surrounding the initiation of these medications in hospital. Furthermore, abrupt cessation of benzodiazepines can cause withdrawal and worsen confusion..$^{22}$

We found that $10 \%$ of patients newly prescribed a $\mathrm{BSH}$ in hospital were discharged with a prescription for a $\mathrm{BSH}$. The accuracy of this is limited by the lack of availability of electronic discharge prescriptions on our surgical wards; however, it is likely an underrepresentation of the true effect given the high rates of PIPs on these wards. Our study highlights the concerning practice of continuing newly prescribed BSH following discharge from hospital.

Sleep disruption and poor quality sleep in hospital is a common issue that leads to significant use of $\mathrm{BSH} .{ }^{15} \mathrm{Non}$ pharmacologic interventions in older adults can be effective in improving sleep quality and reducing the need for $\mathrm{BSH}$; however, they can be time-consuming to implement. ${ }^{23}$ With the exception of preventative strategies used on our Acute Care for Elders unit, formal nonpharmacologic interventions for sleep are not practiced in our hospital. We found that the majority of PIPs were prescribed as sleep aids in the overnight hours. This suggests that disruptions in sleep are leading patients and nursing staff to request pharmacologic treatments and highlights an area with significant room for improvement. Work is underway to 
implement and evaluate safe sleep protocols for older adults.

To our knowledge, we are the first to report an association between training level and PIP of BSH in older adults. The highest rates of PIPs were found among the first-year residents and, after controlling for patient and prescribing event characteristics, such as time of prescription, first-year residents were significantly more likely to prescribe a PIP. First-year residents are more likely to respond first to issues on the wards. There may be pressure on first-year trainees to prescribe sleep aids, as many patients and nurses may seek pharmacologic solutions for symptom management. Knowledge gaps may also be a contributing factor early in their training. A survey of physicians found that residents were more likely than attending physicians to list lack of formal education as a barrier to appropriate prescribing. ${ }^{24}$

Similarities are seen in a study of antibiotic appropriateness, where residents demonstrated gaps in knowledge of treatment of asymptomatic bacteriuria that seemed to vary by specialty. ${ }^{25}$ Interestingly, we found that patients admitted to general internal medicine were prescribed fewer PIPs. This service includes our Acute Care for Elders unit, which is staffed by trained geriatric nurses and other allied health professionals. Residents who rotated on internal medicine are also likely to have received informal teaching about medication safety in older adults. Educational interventions highlighting adverse effects of $\mathrm{BSH}$ and promoting nonpharmacologic solutions should be targeted at first-year residents. However, an interprofessional team approach to sleep disturbance in hospital, in combination with decision support for appropriate BSH use will achieve greater impact than education alone.

Several limitations of this study merit discussion. First, findings from a single academic center may lack generalizability. However, the demographics of our patient population and our rates of BSH use were similar to those reported in previous studies. Second, our study may be subject to observer bias, as the data collectors were not blinded. To minimize this, a strict template and clear appropriateness criteria were developed. Additionally, a second reviewer independently conducted data validation with $100 \%$ agreement among reviewers. Third, we studied prescribing patterns rather than medication administration and lacked data on filling of new $\mathrm{BSH}$ prescriptions in the postdischarge period. However, our primary goal is to determine risk of exposure to a $\mathrm{BSH}$ to minimize it. Fourth, although BSH are discouraged as "first choice for insomnia, anxiety or delirium," ${ }^{4}$ they may be appropriate in limited situations where all nonpharmacologic strategies have failed and patient or staff safety is at risk. In our chart reviews, we were unable to determine whether all nonpharmacologic strategies were exhausted prior to prescription initiation. However, more than $20 \%$ of all PIP were routinely prescribed as part of an admission or postoperative order set, suggesting a reflexive rather than reflective approach to sedative use. Furthermore, the indications of anxiety and agitation were combined as they appear in the CPOE as a combination indication, thus leaving us un- able to determine the true proportion for each indication. However, more than $87 \%$ of all PIPs were for insomnia, reflecting a clear opportunity to improve sleep management in hospital. Last, the lack of a power calculation may have resulted in the study being underpowered and thus affected the ability to detect a significant effect of covariates that have real differences on the likelihood of sedative prescriptions. For example, the low number of prescribing events by second-year residents and staff may have resulted in a type II error when comparing PIP rates with first-year residents.

We found that the majority of newly prescribed $\mathrm{BSH}$ among older adults in hospital were potentially inappropriate. They were most frequently prescribed by first-year residents overnight in response to insomnia. Our findings demonstrate BSH overuse remains prevalent and is associated with poor sleep in hospital. Future work will focus on implementing and evaluating safe sleep protocols and educational interventions aimed at first-year residents.

\section{Acknowledgments}

Elisabeth Pek had full access to all of the data in the study and takes responsibility for the integrity of the data and the accuracy of the data analysis. Ciara Pendrith conducted and is responsible for the statistical analysis.

Disclosure: The authors report no financial conflicts of interest.

\section{References}

1. Glass J, Lanctot KL, Herrmann N, Sproule BA, Busto UE. Sedative hypnotics in older people with insomnia: meta-analysis of risks and benefits. BMJ. 2005;331(7526):1169.

2. Inouye SK. Delirium in older persons. N Engl J Med. 2006;354(11):1157-1165.

3. Morden NE, Colla CH, Sequist TD, Rosenthal MB. Choosing wisely--the politics and economics of labeling low-value services. N Engl J Med. 2014;370(7): 589-592.

4. Ten Things Physicians and Patients Should Question. American Geriatrics Society 2013. Revised April 23, 2015. http://www.choosingwisely.org/societies/american-geriatrics-society/. Accessed April 30, 2016.

5. Five Things Physicians and Patients Should Question. Canadian Geriatrics Society. Released April 2, 2014. http://www.choosingwiselycanada.org/recommendations/geriatrics/. Accessed April 30, 2016.

6. de Groot MH, van Campen JP, Moek MA, Tulner LR, Beijnen JH, Lamoth CJ. The effects of fall-risk-increasing drugs on postural control: a literature review. Drugs Aging. 2013;30(11):901-920.

7. Woolcott JC, Richardson KJ, Wiens MO, et al. Meta-analysis of the impact of 9 medication classes on falls in elderly persons. Arch Intern Med. 2009;169(21): $1952-1960$.

8. Pariente A, Dartigues JF, Benichou J, Letenneur L, Moore N, Fourrier-Réglat A. Benzodiazepines and injurious falls in community dwelling elders. Drugs Aging. 2008;25(1):61-70.

9. Frels C, Williams P, Narayanan S, Gariballa SE. Iatrogenic causes of falls in hospitalised elderly patients: a case-control study. Postgrad Med J. 2002;78(922): 487-489.

10. Pavon JM, Zhao Y, McConnell E, Hastings SN. Identifying risk of readmission in hospitalized elderly adults through inpatient medication exposure. J Am Geriatr Soc. 2014;62(6):1116-1121

11. Kang DY, Park S, Rhee CW, et al. Zolpidem use and risk of fracture in elderly insomnia patients. J Prev Med Public Health. 2012;45(4):219-226.

12. Kolla BP, Lovely JK, Mansukhani MP, Morgenthaler TI. Zolpidem is independently associated with increased risk of inpatient falls. J Hosp Med. 2013;8(1):1-6.

13. Finkle WD, Der JS, Greenland S, et al. Risk of fractures requiring hospitalization after an initial prescription for zolpidem, alprazolam, lorazepam, or diazepam in older adults. J Am Geriatr Soc. 2011;59(10):1883-1890.

14. Elliott RA, Woodward MC, Oborne CA. Improving benzodiazepine prescribing for elderly hospital inpatients using audit and multidisciplinary feedback. Intern Med J. 2001;31(9):529-535.

15. Cumbler E, Guerrasio J, Kim J, Glasheen J. Use of medications for insomnia in the 
hospitalized geriatric population. J Am Geriatr Soc. 2008;56(3):579-581.

16. Somers A, Robays H, Audenaert K, Van Maele G, Bogaert M, Petrovic M. The use of hypnosedative drugs in a university hospital: has anything changed in 10 years? Eur J Clin Pharmacol. 2011;67(7):723-729.

17. Gillis CM, Poyant JO, Degrado JR, Ye L, Anger KE, Owens RL. Inpatient pharmacological sleep aid utilization is common at a tertiary medical center. J Hosp Med. 2014;9(10):652-657

18. Frighetto L, Marra C, Bandali S, Wilbur K, Naumann T, Jewesson P. An assessment of quality of sleep and the use of drugs with sedating properties in hospitalized adult patients. Health Qual Life Outcomes. 2004;2:17

19. Garrido MM, Prigerson HG, Penrod JD, Jones SC, Boockvar KS. Benzodiazepine and sedative-hypnotic use among older seriously Ill veterans: choosing wisely? Clin Ther. 2014;36(11):1547-1554.

20. American Geriatrics Society updated Beers Criteria for potentially inappropriate medication use in older adults: The American Geriatrics Society 2012 Beers Cri- teria Update Expert Panel. J Am Geriatr Soc. 2012;60(4):616-631.

21. Charlson ME, Pompei P, Ales KL, MacKenzie CR. A new method of classifying prognostic comorbidity in longitudinal studies: development and validation. J Chronic Dis. 1987;40(5):373-383.

22. Foy A, Drinkwater V, March S, Mearrick P. Confusion after admission to hospital in elderly patients using benzodiazepines. Br Med J (Clin Res Ed). 1986;293(6554):1072.

23. McDowell JA, Mion LC, Lydon TJ, Inouye SK. A nonpharmacologic sleep protocol for hospitalized older patients. J Am Geriatr Soc. 1998;46(6):700-705.

24. Ramaswamy R, Maio V, Diamond JJ, et al. Potentially inappropriate prescribing in elderly: assessing doctor knowledge, confidence and barriers. J Eval Clin Pract. 2011;17(6):1153-1159.

25. Lee MJ, Kim M, Kim NH, et al. Why is asymptomatic bacteriuria overtreated?: A tertiary care institutional survey of resident physicians. BMC Infect Dis. 2015;15:289. 